\title{
Forbidden subgraphs of power graphs
}

\author{
Pallabi Manna* \\ Department of Mathematics \\ National Institute of Technology Rourkela \\ Rourkela, Odisha - 769008, India \\ mannapallabimath001@gmail.com \\ Peter J. Cameron \\ School of Mathematics and Statistics \\ University of St Andrews \\ North Haugh, St Andrews, Fife, KY16 9SS, U.K. \\ pjc20@st-andrews.ac.uk \\ Ranjit Mehatari ${ }^{\dagger}$ \\ Department of Mathematics \\ National Institute of Technology Rourkela \\ Rourkela, Odisha - 769008, India \\ ranjitmehatari@gmail.com, mehatarir@nitrkl.ac.in
}

Submitted: Oct 20, 2020; Accepted: Jun 5, 2021; Published: Jul 2, 2021

(C) The authors. Released under the CC BY-ND license (International 4.0).

\begin{abstract}
The undirected power graph (or simply power graph) of a group $G$, denoted by $P(G)$, is a graph whose vertices are the elements of the group $G$, in which two vertices $u$ and $v$ are connected by an edge between if and only if either $u=v^{i}$ or $v=u^{j}$ for some $i, j$.

A number of important graph classes, including perfect graphs, cographs, chordal graphs, split graphs, and threshold graphs, can be defined either structurally or in terms of forbidden induced subgraphs. We examine each of these five classes and attempt to determine for which groups $G$ the power graph $P(G)$ lies in the class under consideration. We give complete results in the case of nilpotent groups, and partial results in greater generality. In particular, the power graph is always perfect; and we determine completely the groups whose power graph is a threshold or split graph (the answer is the same for both classes). We give a number of open problems.
\end{abstract} Mathematics Subject Classifications: 05C25.

*Supported by CSIR, India (Grant No-09/983(0037)/2019-EMR-I).

${ }^{\dagger}$ Supported by SERB, India through Core Research Grant (File Number-CRG/2020/000447). 


\section{Introduction}

The study of graph representations is one of the interesting and popular research topic in algebraic graph theory. One of the major graph representation amongst them is the power graphs of finite groups. We found several papers in this context $[2,5,6,9,10,13$, $14,23,26,27,29]$.

Example 1. There are five groups of order 8. The power graph of $C_{8}$ is complete, and the power graph of $\left(C_{2}\right)^{3}$ is a star $K_{1,7}$. The figure shows the power graphs of the other three groups.
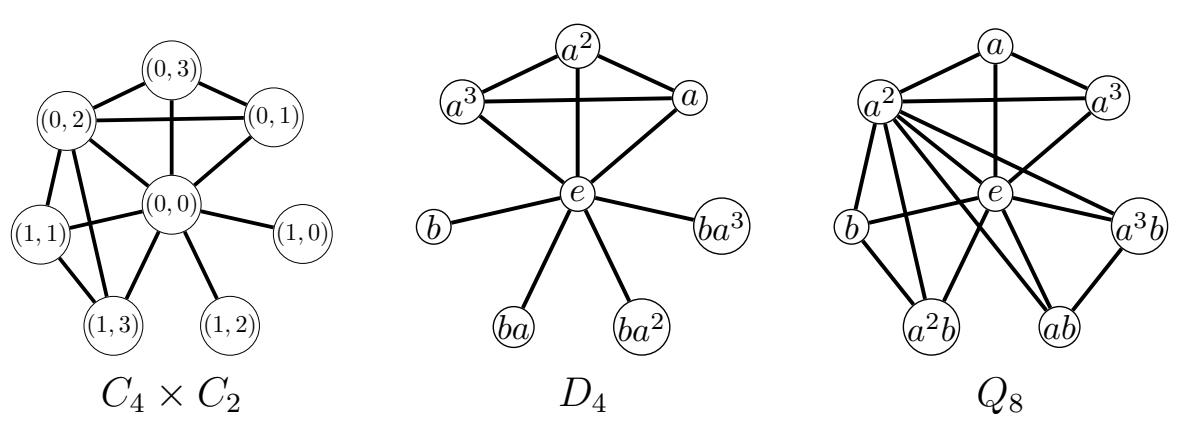

We begin with the history of the concept. The notion of power graphs was first introduced by Kelarev and Quinn [23] in 2002. For a semigroup $S$, the directed power graph of $S$, denoted by $\vec{P}(S)$, is a graph with vertex set $V=S$; for two distinct vertices $u, v \in S$, there exists an $\operatorname{arc}(u, v)$ if $v$ is a power of $u$.

The corresponding undirected graph is called the undirected power graph of $S$, denoted by $P(S)$. The undirected power graph of a semigroup was introduced by Chakrabarty et al. [9] in 2009. So the undirected power graph of $S$ is the graph with vertex set $V(P(S))=S$, with an edge between two vertices $u$ and $v$ if $u \neq v$ and either $v$ is a power of $u$ or $u$ is a power of $v$. In the sequel, "power graph" will mean "undirected power graph".

Later the power graphs of groups were studied. In [9], the authors proved that if $G$ is a finite group then the power graph $P(G)$ is always connected. They further showed that a finite group has a complete undirected power graph if and only if it is cyclic and its order is equal to 1 or $p^{m}$ for some prime $p$. (We give the proof below.) They also counted the number of edges in a power graph of a finite group $G$ by the formula $|E(P(G))|=\frac{1}{2}\left[\sum_{g \in G}(2 o(g)-\phi(o(g))-1)\right]$. The power graph of a finite group is Eulerian if and only if the group $G$ is of odd order [9]. Also $P(G)$ is Hamiltonian if $|G| \geqslant 3$.

In [13], Curtin et al. introduced the concept of proper power graphs. The proper power graph of a group $G$, denoted by $P^{*}(G)$, is the graph obtained from $P(G)$ by deleting the identity. They discussed the diameter of the proper power graph of the symmetric group $S_{n}$ on $n$ symbols. For more information related to proper power graphs we refer to $[13,14,29]$.

In [10], Chattopadhyay et al. gave bounds for the vertex connectivity of the power graph of the cyclic group $C_{n}$. 
In [6], Cameron and Ghosh investigated isomorphism of the power graphs of groups. They showed that if two finite Abelian groups have isomorphic power graphs then they are isomorphic. Moreover they proved that if two finite groups have isomorphic directed power graphs then they have same number of elements of each order. They have also proved that the only finite group $G$ for which $\operatorname{Aut}(G)=\operatorname{Aut}(P(G))$ is the Klein 4-group $V_{4}=C_{2} \times C_{2}$. In [5], Cameron proved that if two finite groups have isomorphic power graphs then they have isomorphic directed power graphs.

Papers dealing with the power graphs of infinite groups include [1, 7, 8, 22, 34, 35]. For more information related to power graphs we refer to the survey article by Abawajy et al. [2].

Let $G=(V, E)$ be any graph and $S$ be any subset of $V$. Then the induced subgraph $G[S]$ is the graph whose vertex set is $S$ and whose edge set consists of all of the edges in $E$ that have both endpoints in $S$. For any graph $H$, the graph $G$ is said to be $H$-free if it has no induced subgraph isomorphic to $H$. The graphs $P_{n}, C_{n}$ and $2 K_{2}$ denote the path on $n$ vertices, the cycle with $n$ vertices, and two disjoint edges with no further edges connecting them.

We use $C_{n}$ for both the cyclic group of order $n$ and the cycle graph on $n$ vertices; the context makes clear in each case which is intended. Also, we use $D_{n}$ for the dihedral group

$$
\left\langle a, b: a^{n}=b^{2}=(a b)^{2}=1\right\rangle
$$

of order $2 n$, rather than for the dihedral group of order $n$ as is often done.

Our general theme is that various important classes of graphs are defined by forbidden induced subgraphs [4,31], and we investigate several of these classes with the aim of determining for which groups $G$ the power graph $P(G)$ belongs to the corresponding class. So we conclude the Introduction with a simple result of this form due to Chakrabarty et al. [9], which illustrates the general problem and will also be used later. Note that a graph is complete if and only if it does not contain the null graph on 2 vertices as an induced subgraph.

Proposition 2. The power graph of a finite group $G$ is complete if and only if $G$ is a cyclic group of prime power order.

Proof. In a cyclic group $G, a$ is a power of $b$ if and only if the order of $a$ divides the order of $b$. If $|G|$ is a power of a prime $p$, then the possible orders are powers of $p$, and so are totally ordered; so $P(G)$ is complete.

Conversely, suppose that $P(G)$ is complete. Then $|G|$ must be a prime power; for, if distinct primes $p$ and $q$ divide $|G|$, then $G$ contains elements $a$ and $b$ of orders $p$ and $q$ respectively, and these are nonadjacent in $P(G)$.

Let $|G|=p^{n}$. Then, for each $i$ with $i \leqslant n$, the number of elements of order $p^{i}$ is at most $\phi\left(p^{i}\right)$, where $\phi$ is Euler's function, since if $a$ and $b$ are two such elements then $b \in\langle a\rangle$, and a cyclic group of order $p^{i}$ contains $\phi\left(p^{i}\right)$ elements of order $p^{i}$. Since

$$
\sum_{i \leqslant n} \phi\left(p^{i}\right)=p^{n}
$$


we see that there are $\phi\left(p^{i}\right)$ elements of order $p^{i}$ for all $i$. In particular, there exist elements of order $p^{n}$; so $G$ is cyclic.

\section{The power graph is perfect}

A finite graph $\Gamma$ is perfect if it has the property that every induced subgraph has clique number equal to chromatic number. We are going to show that every power graph is perfect. This motivates considering subclasses of the class of perfect graphs, as we do in this paper.

The class of perfect graphs is closed under complementation (this is the Weak Perfect Graph Theorem of Lovász [25]), and contains several further important graph classes, including bipartite graphs, line graphs, chordal graphs, interval graphs, and comparability graphs of partial orders. It was shown by Grötschel, Lovász and Schrijver [19] that computational problems which are hard for general graphs (such as graph colouring, maximal clique and maximal independent set) can be solved in polynomial time for perfect graphs using semidefinite programming.

According to the Strong Perfect Graph Theorem, a conjecture of Berge [3] proved by Chudnovsky, Robertson, Seymour and Thomas [11], a graph is perfect if and only if it contains no odd hole or odd antihole as induced subgraph, where an odd hole is an $n$-cycle with $n$ odd and $n \geqslant 5$, and an odd antihole is the complement of an odd hole.

A partial preorder is a binary relation $\rightarrow$ on a set $X$ which is reflexive and transitive. Its comparability graph is the graph on the vertex set $X$ in which $u$ and $v$ are adjacent if and only if either $u \rightarrow v$ or $v \rightarrow u$.

Proposition 3. The comparability graph of a partial preorder is perfect.

Proof. In the case of a partial order, a partial preorder satifying

$$
(x \rightarrow y \text { and } y \rightarrow x) \Rightarrow x=y,
$$

this is one part (the easier part) of Dilworth's Theorem [15]. For completeness, we sketch the proof.

Let $\Gamma$ be the comparability graph of a partial order. A clique in $\Gamma$ is a chain in the partial order. If the largest chain has size $k$, we produce a proper $k$-colouring of the graph as follows: the minimal elements of the partial order form an independent set, to which we assign the first colour, and then remove these vertices. Since every maximal chain contains one of these vertices, the largest chain in the resulting graph has size $k-1$, and so by induction it can be properly coloured with the remaining $k-1$ colours.

Now suppose that $\rightarrow$ is a partial preorder on $X$. Define a relation $\equiv$ on $X$ by the rule

$$
(x \equiv y) \Leftrightarrow(x \rightarrow y \text { and } y \rightarrow x)
$$

Then $\equiv$ is an equivalence relation. Now define a relation $\leqslant$ on the set of equivalence classes by the rule that $[x] \leqslant[y]$ if $x^{\prime} \rightarrow y^{\prime}$ for some (and hence every) $x^{\prime} \in[x]$ and $y^{\prime} \in[y] ;$ then $\leqslant$ is a partial order. 
Now we can refine the partial preorder to a partial order by simply putting a total order on each equivalence class; this does not change the comparability graph, which is thus perfect.

Now we can show that the power graph is a perfect graph. Indeed, we do not require a group; a semigroup will suffice, or indeed any power-associative magma, that is, a set with a binary operation such that the associative law holds for the powers of a single element (so that powers $x^{n}$ can be unambiguously defined).

Proposition 4. The directed power graph of any power-associative magma is a partial preorder.

Proof. This follows immediately from the facts that $x^{1}=x$ and $\left(x^{m}\right)^{n}=x^{m n}$.

Corollary 5. The undirected power graph of a power-associative magma is a perfect graph.

Remark 6. This result holds also for infinite power-associative magmas, if we say that an infinite graph is perfect if every finite induced subgraph has clique number equal to chromatic number.

Remark 7. This satisfactory result shows, for example, that the power graph of a group cannot contain the 5-cycle $C_{5}$ as an induced subgraph (this graph has clique number 2 and chromatic number 3). In the remainder of this paper we look at other examples. But first we pose an open problem concerning two closely related graphs.

The enhanced power graph of a group $G$ is the graph with vertex set $G$, in which $u$ and $v$ are joined if and only if they are both powers of an element $w$. The commuting graph of $G$ is the graph in which $u$ and $v$ are joined if and only if $u v=v u$. It is clear that the power graph is a spanning subgraph of the enhanced power graph, which is itself a spanning subgraph of the commuting graph.

\section{Problem 8.}

(a) For which groups $G$ is the enhanced power graph of $G$ a perfect graph?

(b) For which groups $G$ is the commuting graph of $G$ a perfect graph?

We make two observations about these problems.

Remark 9. The paper of Aalipour et al. [1] contains classifications of groups for which either the enhanced power graph or the commuting graph is equal to the power graph. These groups are examples satisfying the conditions of the problem. So the question is: What other groups can occur?

Remark 10. It is not the case that the enhanced power graph or the commuting graph of every group is perfect. For the commuting graph, we may take $G=S_{5}$, the symmetric group of degree 5; the five transpositions

$$
(1,2),(3,4),(5,1),(2,3),(4,5)
$$


have the property that the induced subgraph is $C_{5}$. For the enhanced power graph, we can work in a larger symmetric group, and take five cycles of pairwise coprime lengths with the same intersection pattern as above, using the fact that two elements of coprime order are joined in the enhanced power graph if and only if they commute.

\section{Cographs}

A graph $G$ is a cograph if it has no induced subgraph isomorphic to the four-vertex path $P_{4}$. Cographs form the smallest class of graphs containing the 1-vertex graph and closed under the operations of disjoint union and complementation.

Cographs have been rediscovered several times and given several different names: for example, Sumner [30] called them heredditary Dacey graphs. The class of cographs contains the threshold graphs that we discuss later, and is included in the class of comparability graphs (they are the comparability graphs of $N$-free partial orders, those obtained from the one-point order by the operations of disjoint union and ordered sum). Thus, cographs are perfect graphs.

In this section, we determine completely the finite nilpotent groups whose power graph is a cograph. We also give a partial analysis of the question for more general groups, using the concept of the prime graph (or Gruenberg-Kegel graph) of a group.

\subsection{Nilpotent groups}

Recall that a finite group $G$ is nilpotent if and only if it is a direct product of its Sylow $p$-subgroups over primes $p$ dividing $|G|$. Note that, in a nilpotent group, elements of different prime orders commute. Our first result determines which finite nilpotent groups $G$ have the property that their power graphs are cographs.

We begin with:

Lemma 11. The power graph of a finite group cannot have an induced 4-vertex path or cycle in which all four vertices are elements whose orders are powers of the same prime p.

Proof. Suppose that $(a, b, c, d)$ is such a path or cycle.

In the directed power graph $\vec{P}(G)$, we cannot have $b \rightarrow c$. For suppose that $b \rightarrow c$. Then $c$ is a power of $b$, and one of $a$ and $b$ is a power of the other; so $a, b, c$ are all contained in a cyclic $p$-group. But the power graph of a cyclic $p$-group is complete, by Proposition 2 , and so there is an edge $\{a, c\}$, contrary to assumption.

By the same argument applied to $(b, c, d)$, we cannot have $c \rightarrow b$. Therefore $b$ and $c$ are not joined in the power graph, a contradiction.

Theorem 12. Let $G$ be a finite nilpotent group. Then $P(G)$ is a cograph if and only if either $|G|$ is a prime power, or $G$ is cyclic of order $p q$ for distinct primes $p$ and $q$.

Proof. Lemma 11 shows that if $G$ is a $p$-group with $p$ prime, then $P(G)$ contains no induced path of length 3 , and so it is a cograph. 
Now we show that $G=C_{p q}$ is a cograph if $p$ and $q$ are distinct primes. In any group, two elements $a, b$ satisfy $a \rightarrow b$ and $b \rightarrow a$ if and only if $\langle a\rangle=\langle b\rangle$; in a cyclic group, this is equivalent to the condition that $a$ and $b$ have the same order, and hence the same neighbours. So if $(a, b, c, d)$ is an induced path, then no two of $a, b, c, d$ have the same order. Hence one of them is the identity. But the identity is joined to all other vertices, a contradiction.

Conversely, let $G$ be a finite nilpotent group whose power graph is a cograph. Suppose first that three primes $p, q, r$ divide $|G|$. Let $a, b, c$ be elements with orders $p, q, r$ respectively. These three elements commute pairwise; so $a b$ and $b c$ have orders $p q$ and $q r$ respectively. Then $(a, a b, b, b c)$ is an induced path, a contradiction.

So suppose that only two primes $p$ and $q$ divide $|G|$. Then $G=P \times Q$ where $P$ is a $p$-group and $Q$ a $q$-group.

Suppose first that $P$ is not cyclic. Then there are elements $a, b \in P$ which are not adjacent in the power graph. If $c$ is a non-identity element of $Q$, then $(a, a c, c, b c)$ is an induced path in $P(G)$. So $P$ (and similarly $Q$ ) is cyclic.

Now suppose that $|P|>p$. If $a$ is an element of order $p^{2}$ in $P$, and $b$ an element of order $q$ in $Q$, then $\left(a, a^{p}, a^{p} b, b\right)$ is an induced path in $P(G)$. (These elements have orders $p^{2}, p, p q, q$ respectively; so non-consecutive elements are not joined.)

So $|P|=p$, and similarly $|Q|=q$; thus $G=C_{p} \times C_{q}=C_{p q}$.

\subsection{The prime graph, or Gruenberg-Kegel graph}

Suppose that $G$ is a group which contains no element whose order is the product of two primes. Then every element of $G$ has prime power order, and every edge of the power graph joins elements of the same prime power order. Now Lemma 11 shows that $P(G)$ contains no induced $P_{4}$, and so it is a cograph

These considerations lead us to the notion of the prime graph $\Pi(G)$ of the group $G$. The vertices of $\Pi(G)$ are the prime divisors of $|G|$; there is an edge joining $p$ to $q$ if $G$ contains an element of order $p q$. The argument in the previous paragraph shows:

Theorem 13. Let $G$ be a group whose prime graph is a null graph. Then $P(G)$ is a cograph.

The prime graph was introduced by Gruenberg and Kegel in an unpublished manuscript studying integral representations of groups in 1975. (The prime graph is often called the Gruenberg-Kegel graph.) They observed that there are very strong restrictions on the structure of a group whose prime graph is disconnected. Subsequently, Williams [33] (a student of Gruenberg) published their theorem. With a small addition, it states the following. Here a 2-Frobenius group is a group with normal subgroups $H$ and $K$ with $K \leqslant H$ such that

(a) $H$ is a Frobenius group with Frobenius kernel $K$;

(b) $G / K$ is a Frobenius group with Frobenius kernel $H / K$. 
The symmetric group $S_{4}$ is an example, with $H=A_{4}$ and $K=V_{4}$.

Moreover, suppose that $G$ has even order. Let $\pi_{1}$ be the set of primes which are vertices of the connected component containing the prime 2 . A $\pi_{1}$-group denotes a group whose order is divisible only by primes in $\pi_{1}$.

Theorem 14. The prime graph of $G$ is disconnected only if $G$ satisfies one of the following:

(a) $G$ is Frobenius or 2-Frobenius;

(b) $|G|$ is even, and $G$ is an extension of a nilpotent $\pi_{1}$-group by a simple group by a $\pi_{1}$-group.

Williams observed that, in the second case of the theorem, the simple group itself must have disconnected prime graph, and he analysed the simple groups to find which have disconnected prime graph. The results cannot be summarised here since they comprise extended tables. The tables contained some errors which were corrected by Kondrat'ev and Mazurov [24].

The only simple groups with the property that the prime graph is a null graph are the alternating groups $A_{5}$ and $A_{6}$ and the groups $\operatorname{PSL}(2,7), \operatorname{PSL}(2,8), \operatorname{PSL}(2,17), \operatorname{PSL}(3,4)$, and $\mathrm{Sz}(8)$. So these groups have power graphs which are cographs.

In the other direction, Williams observed that, if $G$ is a non-solvable group with disconnected prime graph and $\pi$ is the vertex set of a connected component not containing 2 , then $G$ has a nilpotent Hall $\pi$-subgroup which contains the centraliser of each of its elements. By our earlier result, we obtain the following.

Theorem 15. Let $G$ be a non-solvable group whose power graph is a cograph, and suppose that the prime graph of $G$ is disconnected. Then, with possibly one exception, each component of the prime graph is either an isolated vertex or an isolated edge joining two primes which divide $|G|$ to the first power only (the exception, if any, is the component containing 2).

Information about the simple groups satisfying this condition can be obtained from the results of $[33,24]$. However, some problems remain. We give an example. For which prime powers $q$ is power graph of the group $\operatorname{PSL}(2, q)$ a cograph? If $q$ is even, then the prime graph is the union of three complete graphs, on the sets of prime divisors of $q, q-1$ and $q+1$; if $q$ is odd, we replace $q-1$ and $q+1$ by $(q-1) / 2$ and $(q+1) / 2$.

(a) Let $q=2^{n}$. If $n$ is even, say $n=2 m$ with $m>1$, then $2^{2 m}-1=\left(2^{m}-1\right)\left(2^{m}+1\right)$, and the two factors cannot both be primes except when $m=2$; so $q=4$ and $q=16$ are the only examples. If $n$ is odd, then we require that $2^{n}-1$ has at most two prime divisors; also $3 \mid 2^{n}+1$, so we require that $\left(2^{n}+1\right) / 3$ is prime. Examples include $q=8,32,128,2048,8192, \ldots$

(b) Let $q$ be a power of a prime $p>3$. Then one of $(q-1) / 2$ and $(q+1) / 2$ is even, and one is divisible by 3 . So there are several possibilities: 
- $(q-1) / 2=2 r,(q+1) / 2=3 s$, or $(q-1) / 2=3 s,(q+1) / 2=2 r$, with $r$ and $s$ primes (possible values of $q$ include $27,43,67, \ldots$ );

- one of $(q-1) / 2$ and $(q+1) / 2$ is a power of 2 , the other is three times a prime (examples $q=31,257, \ldots$ );

- one of $(q-1) / 2$ and $(q+1) / 2$ is a power of 3 , the other is twice a prime (examples $q=19,53,163, \ldots$ );

- one of $(q-1) / 2$ and $(q+1) / 2$ is a power of 2 , the other a power of 3 (by the solution of Catalan's equation, this holds only for $q=5,7$ and 17).

(c) For $q$ a power of 3, a similar analysis is possible. Examples include $q=9,27,243$, $2187, \ldots$

Problem 16. Are there infinitely many prime powers $q$ such that the power graph of $\operatorname{PSL}(2, q)$ is a cograph?

\section{Chordal graphs}

A graph $\Gamma$ is chordal if it contains no induced cycles of length greater than 3 ; in other words, every cycle on more than 3 vertices has a chord.

Chordal graphs also arise in several different contexts: they are the intersection graphs of subtrees of a tree, or the graphs with a perfect elimination order [17]. The class includes the split graphs and is contained within the class of perfect graphs.

In this section we determine which finite nilpotent groups have chordal power graphs. We already know that power graphs contain no odd cycles of length greater than 3 .

Theorem 17. If $G$ is a group of prime power order then $P(G)$ is chordal.

Proof. We saw in Lemma 11 that $P(G)$ has no induced path of length 3, and hence no induced even cycle of length 6 or greater. The same lemma also shows that there is no induced 4-cycle.

Lemma 18. Let $G$ be a group whose order is a power of a prime $p$. Suppose that there is no induced path of length 2 in $P(G)$ not containing the identity. Then either $G$ is cyclic or $G$ has exponent $p$. Conversely, if $G$ is cyclic or has exponent $p$, then $P(G) \backslash\{1\}$ contains no induced path of length 2.

Proof. If $G$ is cyclic then $P(G)$ is complete; if $G$ has exponent $p$, then $P(G)$ consists of complete graphs of order $p$ with a single vertex 1 in common. So the converse holds.

So assume that $G$ is a $p$-group and $P(G)$ has no induced path of length 2 not containing the identity; assume for a contradiction that $G$ is neither cyclic nor of exponent $p$.

First observe that $G$ cannot contain a subgroup $C_{p^{2}} \times C_{p}$. For if $a$ and $b$ were generators of the factors in such a subgroup, then $\left(a, a^{p}, a b\right)$ would be a path of length 2 . In particular, the centre of $G$ is elementary abelian. If $Z(G)$ is not cyclic, then choose an element $a$ of order $p^{2}$ in $G$, and an element $b \in Z(G) \backslash\langle a\rangle$; then $\langle a, b\rangle \cong C_{p^{2}} \times C_{p}$, a contradiction. 
Thus $Z(G) \cong C_{p}$. This argument also shows that, if $a$ is any element of $G$ of order greater than $p$, then some power of $a$ generates $Z(G)$.

Suppose that $b$ is an element of order $p$ not in $Z(G)$, and $a$ an element of order $p^{2}$, so that $a^{p} \in Z(G)$. If $a$ and $b$ commute, then they generate $C_{p^{2}} \times C_{p}$; so suppose not. Then $\left(b^{-1} a b\right)^{p}=a^{p}$. If $b^{-1} a b \notin\langle a\rangle$, then $\left(a, a^{p}, b^{-1} a b\right)$ is a path of length 2 ; so suppose that $b^{-1} a b \in\langle a\rangle$. Then $\langle a, b\rangle$ is a non-abelian subgroup of order $p^{3}$ and exponent $p^{2}$, and we may suppose that $b^{-1} a b=a^{p+1}$. Now we can compute that $(a b)^{p}=a^{p}$, and so $\left(a, a^{p}, a b\right)$ is a path of length 2 .

Theorem 19. Let $G$ be a finite nilpotent group which is not of prime power order. Then $P(G)$ is chordal if and only if $|G|$ has two prime divisors, one of the two Sylow subgroups is cyclic, and the other has prime exponent.

Proof. If there are three prime divisors of $|G|$, say $p, q, r$, let $a, b, c$ be elements of these orders. Then $(a, a b, b, b c, c, c a, a)$ is an induced 6-cycle in $P(G)$.

Suppose that $p$ and $q$ are the only prime divisors of $|G|$, and $P, Q$ the corresponding Sylow subgroups. Suppose that $P$ is not cyclic. If also $Q$ is not cyclic, then choose $a, b$ nonadjacent vertices in $P$ and $c, d$ nonadjacent vertices in $Q$; then $(a, a c, c, b c, b, b d, d, a d, a)$ is an induced 8-cycle. So $Q$ is cyclic. If $(a, b, c)$ is an induced path of length 2 on nonidentity elements of $P$, and $d$ a non-identity element of $Q$, then $b$ is a power of $a$ and a power of $c$ (but not the reverse) and so $(b, a d, d, c d)$ is an induced 4-cycle in $P(G)$. By Lemma 18, $P$ has prime exponent.

Now $P\left(C_{p^{2}} \times C_{q^{2}}\right)$ is not chordal, since (if the factors are generated by $a$ and $b$ ) it contains the cycle

$$
\left(\left(1, b^{q}\right),\left(a^{p}, b\right),\left(a^{p}, 1\right),\left(a, b^{q}\right)\right) .
$$

So $P$ and $Q$ are not both cyclic of composite order; thus one is cyclic and the other has prime exponent.

Conversely, let $H$ be a group of exponent $p$, and $G=H \times C_{q^{\beta}}$, and suppose that $P(G)$ contains a cycle of even length. Then $P(H)$ consists of complete graphs of size $p$ sharing only the identity. Any two vertices of the form $(1, y)$ in $G$ are adjacent; so the cycle contains at most two such vertices, and if two then they must be consecutive. The remaining vertices all have the form $(h, y)$ with $h \neq 1$. Then for two consecutive vertices $(h, y)$ and $\left(h^{\prime}, y^{\prime}\right)$, we must have $\langle h\rangle=\left\langle h^{\prime}\right\rangle$, and we may without loss of generality assume that $h=h^{\prime}$. So the entire cycle is contained in $C_{p} \times C_{q^{\beta}}$, and it suffices to show that the power graph of this group is chordal. The argument above shows that the length of an even cycle cannot be greater than 4 .

So finally, let $\left(\left(1, y_{1}\right),\left(1, y_{2}\right),\left(a, y_{3}\right),\left(a, y_{4}\right)\right)$ be a 4 -cycle. Let the order of $y_{i}$ be $q^{m_{i}}$. Then we have

$$
m_{1}>m_{3} \geqslant m_{2}>m_{4} \geqslant m_{1},
$$

since, for example, $y_{1}$ is a power of $y_{4}$ but is not a power of $y_{3}$. But this is impossible.

Remark 20. A group of exponent 2 is abelian, and so is a direct product of cyclic groups of order 2. But there are non-abelian groups of exponent $p$ for any odd prime $p$, for example

$$
G=\left\langle a, b, z: a^{p}=b^{p}=z^{p}=1,[a, b]=z,[a, z]=[b, z]=1\right\rangle
$$


of order $p^{3}$.

\section{$5 \quad$ Threshold graphs and split graphs}

A threshold graph is a graph containing no induced subgraph isomorphic to $P_{4}, C_{4}$ or $2 K_{2}[12,21,28]$. Thus every threshold graph is a cograph. Threshold graphs form the smallest family of graphs containing the one-vertex graph and closed under the operations of adding an isolated vertex and adding a vertex joined to all others.

Applications of threshold graphs in computer science and psychology can be found in $[12,21]$.

An $n$-vertex threshold graph is can be represented by a binary string $a_{1} a_{2} \cdots a_{n}$ where $a_{1}=0$ and, for $2 \leqslant a_{i} \leqslant n, a_{i}=0$ if the vertex $i$ is added as an isolated vertex, and $a_{i}=1$ if it is added as a vertex joined to all other existing vertices. This sequence gives a simple representation of the graph.

A graph $G$ is split if the vertex set is the disjoint union of two subsets $A$ and $B$ such that $A$ induces a complete graph and $B$ a null graph. Split graphs were introduced independently by several authors, first by Földes and Hammer [16], and are easy to recognise algorithmically (in particular, they can be recognised by their degree sequences).

A graph is split if and only if it contains no induced subgraph isomorphic to $C_{4}, C_{5}$ or $2 K_{2}$.

A common feature of these two classes is that both exclude $2 K_{2}$. In [26], Ma and Feng proved that power graph of a finite group is a split graph if and only is it does not contains $2 K_{2}$. In fact we observe that the same is true for threshold graph. In this section we are going to determine all finite groups whose power graph excludes $2 K_{2}$.

We say that the finite group $G$ satisfies the intersection condition (IC) if $G$ does not contain subgroups $H$ and $K$ such that both $H \backslash K$ and $K \backslash H$ contain elements of order greater than 2 .

Theorem 21. For a finite group $G$, the following conditions are equivalent:

(a) $P(G)$ is a threshold graph;

(b) $P(G)$ is a split graph;

(c) $P(G)$ contains no induced subgraph isomorphic to $2 K_{2}$;

(d) $G$ satisfies the intersection condition;

(e) $G$ is cyclic of prime power order, or an elementary abelian or dihedral 2-group, or cyclic of order $2 p$, or dihedral of order $2 p^{n}$ or $4 p$, where $p$ is an odd prime.

Proof. We noted already that each of (a) and (b) implies (c).

Next we show that (c) implies (d). If IC fails, let $H$ and $K$ be subgroups of $G$ and $x \in H \backslash K, y \in K \backslash H$, where $x$ and $y$ are elements with order greater than 2 . Then $\left\{x, x^{-1}, y, y^{-1}\right\}$ induces $2 K_{2}$ (with edges $\left\{x, x^{-1}\right\}$ and $\left\{y, y^{-1}\right\}$ only).

Now we classify groups satisfying IC. First suppose that $G$ has prime power order $p^{n}$. If $p$ is odd, then $G$ has at most one subgroup of each order $p^{i}$ with $i<n$, which forces 
$G$ to be cyclic. So suppose that $p=2$. Let $H$ be a cyclic subgroup of $G$ of maximum order. If $H=G$, then $G$ is cyclic; if $|H|=2$, then $G$ is elementary abelian. By IC, every element outside $H$ has order 2, which implies that $G$ is dihedral.

Now suppose that $G$ does not have prime power order. By IC, $|G|$ is divisible by only one odd prime (since two Sylow subgroups of odd prime power order would violate IC). Let $p$ be an odd prime divisor of $|G|$, and $P$ a Sylow $p$-subgroup of $G$. Then $P$ is the unique Sylow $p$-subgroup of $G$, and so is normal. Let $T$ be a Sylow 2-subgroup. If $T$ contains an element $x$ of order greater than 2 , then $\langle x\rangle$ and $P$ violate IC; so $T$ is elementary abelian. Also $P$ is cyclic, and its automorphism group is therefore cyclic; so $T$ induces an automorphism group of $P$ of order 1 or 2 , so $\left|C_{T}(P)\right|$ is a subgroup of index at most 2 in $P$.

If $T$ contained distinct elements $s$ and $t$ centralising $P$, then $\langle s, P\rangle$ and $\langle t, P\rangle$ violate IC. Also, if $1 \neq t \in C_{T}(P)$ and $|P|>p$, choose an element $x$ of order $p^{2}$ in $P$; then $\langle x\rangle$ and $\left\langle t, x^{p}\right\rangle$ violate IC.

So we are left with three cases:

- $|P|=2$ and $C_{T}(P)=1$. Then the non-identity element of $T$ inverts $P$, and $G$ is dihedral of order $2 p^{n}$, where $|P|=p^{n}$.

- $|P|=2$ and $C_{T}(P)=P$. Then $|P|=p$ and $G$ is cyclic of order $2 p$.

- $|P|=4$ and $\left|C_{T}(P)\right|=2$. Then $G=C_{2} \times D_{p}=D_{2 p}$.

The final step is to show that the groups $G$ in (e) have the property that $P(G)$ is threshold and split, that is, contains no induced $P_{4}, C_{4}, 2 K_{2}$ or $C_{5}$. Here $C_{5}$ is excluded since $P(G)$ is perfect (Corollary 5 ). We note also that, if $H$ is a graph having no isolated vertex and no vertex joined to all others, and $P\left(C_{n}\right)$ is $H$-free, then also $P\left(D_{n}\right)$ is $H$-free. For $P\left(D_{n}\right)$ consists of $P\left(C_{n}\right)$ with $n$ further vertices joioned only to the identity (which is joined to all vertices of $\left.P\left(C_{n}\right)\right)$, so any induced copy of $H$ would have to be contained in $P\left(C_{n}\right) \backslash\{1\}$.

Thus we only have to deal with cyclic groups. Now if $G$ is cyclic of prime power order, then $P(G)$ is complete; and if $G$ is cyclic of order $2 p$, then the complement of $P(G)$ is a star (on the elements of orders 2 and $p$ ) together with $p$ isolated vertices (the identity and the generators). All these graphs exclude $P_{4}, C_{4}$ and $2 K_{2}$ (a self-complementary collection of graphs).

\section{References}

[1] G. Aalipour, S. Akbari, P. J. Cameron, R. Nikandish, and F. Shaveisi. On the structure of the power graph and the enhanced power graph of a group. Electron. $J$. Combin., 24(3):\#P3.16, 2017.

[2] J. Abawajy, A. Kelarev, and M. Chowdhury Power graphs: A survey. Electron. J. Graph Theory Appl., 1(2):125-147, 2013. 
[3] C. Berge. Perfect graphs. Six Papers on Graph Theory. Indian Statistical Institute, Calcutta, 1963, pp. 1-21.

[4] A. Brandstädt, V. B. Le, and J. P. Spinrad. Graph Classes: A Survey. Society for Industrial and Applied Mathematics, Philadelphia, PA, USA, 1999.

[5] P. J. Cameron. The power graph of finite group II. Journal of Group Theory, 13(6):779-783, 2010.

[6] P. J. Cameron and S. Ghosh. The power graph of a finite group. Discrete Math., 311(13):1220-1222 2011.

[7] P. J. Cameron, H. Guerra, and Š. Jurina. The power graph of a torsion-free group. J. Algebraic Combinatorics, 49:83-98, 2019.

[8] P. J. Cameron and S. H. Jafari. On the connectivity and independence number of power graphs of groups. Graphs and Combinatorics, 36:895-904, 2020.

[9] I. Chakrabarty, S. Ghosh, and M. K. Sen. Undirected power graphs of semigroups. Semigroup Forum, 78:410-426, 2009.

[10] S. Chattopadhyay, K. L. Patra, and B. K. Sahoo. Vertex connectivity of the power graph of a finite cyclic group. Discrete Applied Mathematics, 266:259-271, 2019.

[11] M. Chudnovsky, N. Robertson, P. Seymour, and R. Thomas. The strong perfect graph theorem. Annals of Mathematics, 164:51-229, 2006.

[12] V. Chvátal and P. L. Hammer. Aggregations of inequalities in integer programming. Ann. Discrete Math., 1:145-162, 1977.

[13] B. Curtin, G. R. Pourgholi, and H. Yousefi-Azari. On the punctured power graph of a finite group. Australasian Journal of Combinatorics, 62(1):1-7, 2015.

[14] A. Doostabadi, M. Farrokhi, and D. Ghouchan. On the connectivity of proper power graph of finite groups. Communications in Algebra, 43(10):4305-4319, 2015.

[15] R. P. Dilworth. A decomposition theorem for partially ordered sets. Ann. Math., 51(1):161-166, 1950.

[16] S. Földes and P. L. Hammer. Split graphs having Dilworth number two. Canad. J. Math., 29(3):666-672, 1977.

[17] D. R. Fulkerson and O. A. Gross. Incidence matrices and interval graphs. Pacific J. Math., 15(3):835-855, 1965.

[18] D. Gorenstein and J. H. Walter. On finite groups with dihedral Sylow 2-subgroups. Illinois J. Math. 6(4):553-593, 1962.

[19] M. Grötschel, L. Lovász, and A. Schrijver. Geometric Algorithms and Combinatorial Optimization. Springer-Verlag, Berlin, 1988.

[20] Marshall Hall, Jr.. The Theory of Groups. Macmillan, New York, 1959.

[21] P. B. Henderson and Y. Zalcstein. A graph-theoretic characterization of the PV class of synchronizing primitives. SIAM J.Scientific Computing 6(1):88-108, 1977.

[22] S. H. Jafari. A description of automorphism group of power graphs of finite groups. arXiv: 1902.05323, 2019. 
[23] A. Kelarev and S. J. Quinn. Directed graphs and combinatorial properties of semigroups. J. Algebra, 251(1):16-26, 2002.

[24] A. S. Kondrat'ev and V. D. Mazurov. Recognition of alternating groups of prime degree from the orders of their elements. Siberian Math. J., 41(2):294-302, 2000.

[25] L. Lovász. Normal hypergraphs and the perfect graph conjecture. Discrete Mathematics, 2(3):253-267, 1972.

[26] X. Ma and M. Feng. On the chromatic number of the power graph of a finite group. Indag. Math. (NS), 26(4): 626-633, 2015.

[27] X. Ma and H. Su. On the order supergraph of the power graph of a finite group. Ricerche mat., 2020. https://doi.org/10.1007/s11587-020-00520-w

[28] V. N. Mahadev and U. N. Peled. Threshold Graphs and Related Topics, Elsevier, 1995.

[29] K. Pourghobadi and S. H. Jafari. The diameter of power graphs of symmetric groups. J. Algebra Appl., 17(12):1850234, 11pp, 2018.

[30] D. P. Sumner. Dacey graphs. J. Aust. Math. Soc., 18(4):492-502, 1974.

[31] N. Trotignon. Perfect graphs, in: L.W. Beineke, R.J. Wilson (Eds.), Topics in Chromatic Graph Theory, Cambridge University Press, 2015.

[32] J. H. Walter. The characterization of finite groups with abelian Sylow 2-subgroups. Ann. Math., 89(3):405-514, 1969.

[33] J. S. Williams. Prime graph components of finite groups. J. Algebra, 69:487-513, 1981.

[34] S. Zahirović. The power graph of a torsion-free group of nilpotency class 2. arXiv:1911.00555, 2019.

[35] S. Zahirović. The power graph of a torsion-free group determines the directed power graph. arXiv:2006.01984, 2020. 